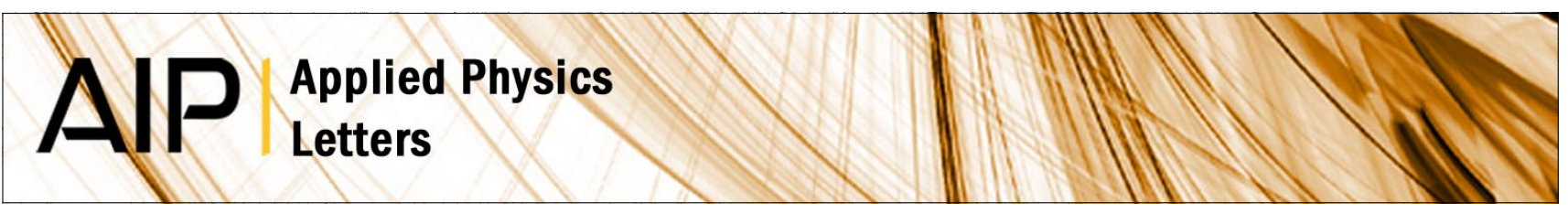

\title{
Sign reversal in the dielectric anisotropy as functions of temperature and frequency in the nematic phase of a bent-core mesogen
}

Yun Jang, Vitaly P. Panov, C. Keith, C. Tschierske, and J. K. Vij

Citation: Appl. Phys. Lett. 97, 152903 (2010); doi: 10.1063/1.3498674

View online: http://dx.doi.org/10.1063/1.3498674

View Table of Contents: http://apl.aip.org/resource/1/APPLAB/v97/i15

Published by the American Institute of Physics.

\section{Related Articles}

Polarizable model of water with field-dependent polarization

J. Chem. Phys. 135, 234110 (2011)

Sign reversal of dielectric anisotropy of ferroelectric liquid crystals doped with cadmium telluride quantum dots Appl. Phys. Lett. 99, 072902 (2011)

Room temperature ferromagnetism in $\mathrm{ZnO}$ prepared by microemulsion AIP Advances 1, 032127 (2011)

A stable and switchable uniform lying helix structure in cholesteric liquid crystals Appl. Phys. Lett. 99, 041108 (2011)

Analysis of a static undulation on the surface of a thin dielectric liquid layer formed by dielectrophoresis forces J. Appl. Phys. 110, 024107 (2011)

\section{Additional information on Appl. Phys. Lett.}

Journal Homepage: http://apl.aip.org/

Journal Information: http://apl.aip.org/about/about_the_journal

Top downloads: http://apl.aip.org/features/most_downloaded

Information for Authors: http://apl.aip.org/authors

\section{ADVERTISEMENT}

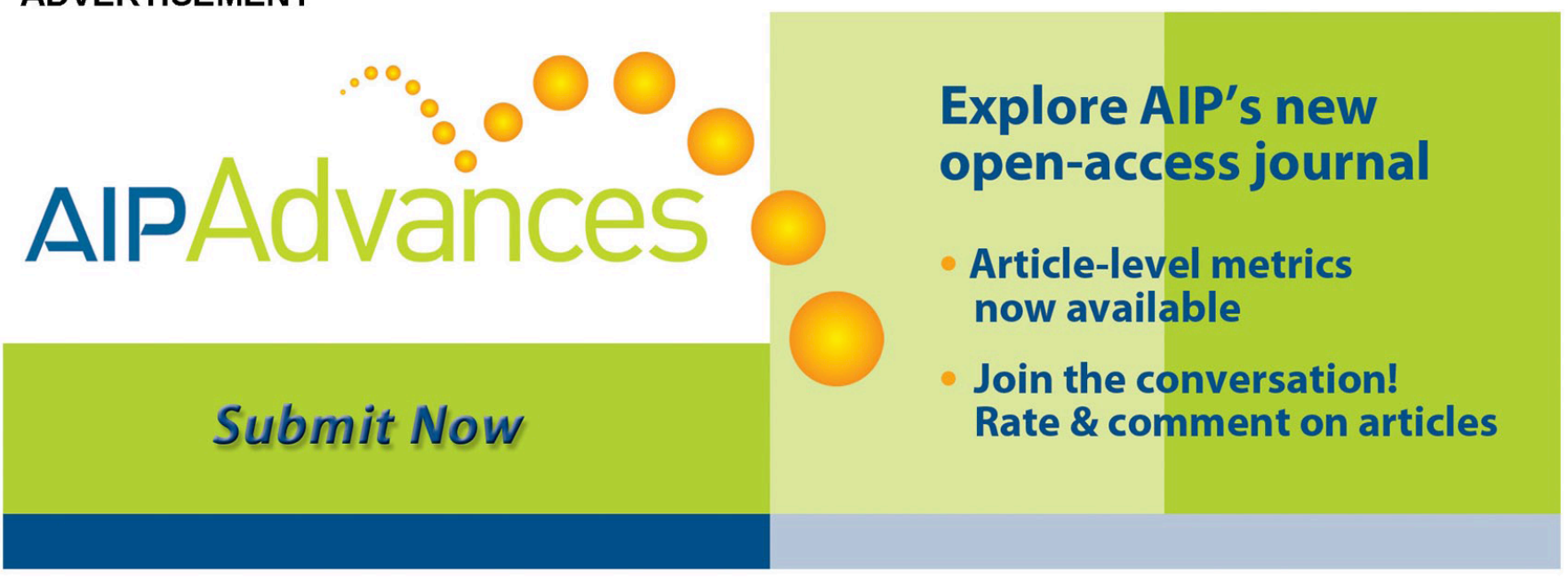




\title{
Sign reversal in the dielectric anisotropy as functions of temperature and frequency in the nematic phase of a bent-core mesogen
}

\author{
Yun Jang, ${ }^{1}$ Vitaly P. Panov, ${ }^{1}$ C. Keith, ${ }^{2}$ C. Tschierske, ${ }^{2}$ and J. K. Vij ${ }^{1, a)}$ \\ ${ }^{1}$ Department of Electronic and Electrical Engineering, Trinity College, University of Dublin, Dublin 2, \\ Ireland \\ ${ }^{2}$ Institute of Organic Chemistry, Martin-Luther-University Halle-Wittenberg, D-06120 Halle,Germany
}

(Received 11 May 2010; accepted 16 September 2010; published online 12 October 2010)

\begin{abstract}
The sign reversal in the dielectric anisotropy $\left(\epsilon_{\|}^{\prime}-\epsilon_{\perp}^{\prime}\right)$ from positive to negative is observed for 4-cyanoresorcinol bisbenzoate system with temperature and frequency in the nematic phase of a bent-core system as the temperature is reduced. This cross-over in dielectric anisotropy is such that at temperatures much below the cross-over temperature, the negative anisotropy is found to be independent of frequency. The experimental observation is supported by results from optical transmittance spectroscopy. This observation is suggested to arise from the following two sources: the presence of cybotactic clusters found by $\mathrm{x}$-ray scattering in the same compound in its nematic phase and conformers that may change the bent core angle at a certain temperature as observed by nuclear magnetic resonance in a similar compound. (C) 2010 American Institute of Physics.
\end{abstract}

[doi:10.1063/1.3498674]

The dielectric response of liquid crystals (LCs) is one of the most important characteristic physical properties required for numerous applications in electro-optics. The director reorientation in the electric field, known as the Freederick's transition, is dependent on the dielectric anisotropy. The latter thus plays an important role in the operation of LC displays. The sign of $\Delta \epsilon^{\prime}$ in LCs could be positive or negative and the amplitude in some cases shows the sign reversal as a function of frequency. In some studies this is also labeled as the dual frequency nematic (DFN) LC mode ${ }^{1,2}$ and is successfully exploited in the addressing schemes for increasing the switching speed of nematics. In the DFN mode, $\Delta \epsilon^{\prime}$ is positive and negative for low and high frequencies, respectively; the frequency for which $\Delta \epsilon^{\prime}=\left(\epsilon_{\|}^{\prime}-\epsilon_{\perp}^{\prime}\right)$ changes sign from positive to negative is called the crossover frequency $f_{c}$. On the logarithmic plot, $f_{c}$ was initially found to be linearly related to the inverse of absolute temperature, ${ }^{1}$ though nonlinearity has also been recently observed. ${ }^{3}$ This phenomenon is normally associated with the restrictions in the molecular rotation around the short axis. For frequencies higher than a few decades of kilohertz, the rotation around the short axis is too sluggish to respond to the electric field. This in turn leads to a reduction in the component of the dipole moment parallel $\mu_{\|}$to the long molecular axis which can respond to the electric field applied across the cell. On the contrary, the rotation around the long axis is relatively free and in most cases of calamitic LCs continues on to a much higher frequency of even a few gigahertz. The phenomena in bent-core systems could be different for following two reasons: the presence of cybotactic clusters in the nematic phase and the possibility that the existence of a number of molecular conformations may change the net dipole moments parallel $\mu_{\|}$and perpendicular $\mu_{\perp}$ to the long molecular axis as well the bent-core angle. In this letter, we present results of the sign reversal of $\Delta \epsilon^{\prime}$ as functions of both frequency and temperature in the nematic phase of a bent-core compound as

${ }^{a)}$ Electronic mail: jvij@tcd.ie. well present the data for $\epsilon_{\|}^{\prime}, \epsilon_{\perp}^{\prime}$, and that for the dielectric anisotropy for different frequencies.

For LCs with calamitic mesogens, $\Delta \epsilon^{\prime}$ is governed mainly by the dipole moment of the polar groups in the mesogen, the latter is generally rigid. However for the bentcore molecules due to the presence of a number of polar groups in the molecule, the calculation of the total dipole moment is extremely complex arising from a possibility of the existence of a range of conformations that become possible due to the rotation of groups around bonds. ${ }^{4}$ In addition, complex steric interactions in the bent-core systems are present, the nature of which may change with temperature. ${ }^{5}$

The material under investigation (shown in Fig. 1, C7-BAN-CN) synthesized in Halle is one of the 4-cyanoresorcinol bisbenzoates. ${ }^{6}$ Evidence from x-ray scattering suggests that $\mathrm{SmC}$-type $\left(\mathrm{N}_{\mathrm{CybC}}\right)$ clusters are present in its nematic phase. The dielectric response is investigated in both homeotropic and planar cells using broadband high resolution dielectric spectrometer (Novocontrol GmbH, Germany). Dielectric/optical cells, each of $8 \mu \mathrm{m}$ sample thickness, were prepared by using two low resistance indium tin oxide glass plates $(20 \Omega / \square)$. The coating agent depends on the type of the alignment/cell configuration to be achieved. The aligning agents RN-1175 (Nissan Chemical Co. ) and AL60702 (JSR Co. Japan) are used for planar and homeotropic cells, respectively,

Dielectric spectra are measured for cells where the LC molecules are aligned in planar and homeotropic configurations. These yield perpendicular $\epsilon_{\perp}^{\prime}$ and parallel dielectric $\epsilon_{\|}^{\prime}$ permittivities, respectively. The parallel components of the

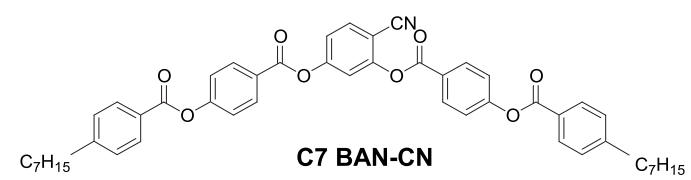

FIG. 1. Chemical structure of C7-BAN-CN. Transition temperatures: $\mathrm{Cr} 96$ $(\mathrm{SmC} 25 \mathrm{CybC} 41) \mathrm{N}_{\mathrm{CybC}} 111$ Iso $\left(\mathrm{T} /{ }^{\circ} \mathrm{C}\right.$, values in brackets refer to monotropic transitions as observed under cooling, see Ref. 6). 

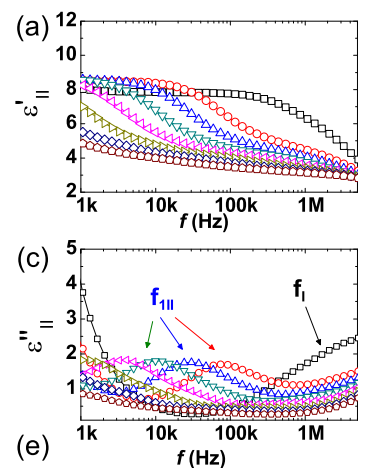

(e)

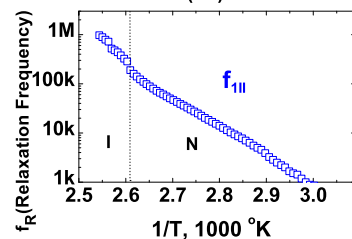

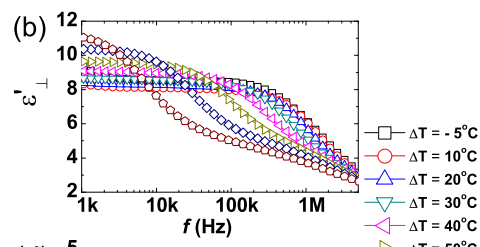

(d)
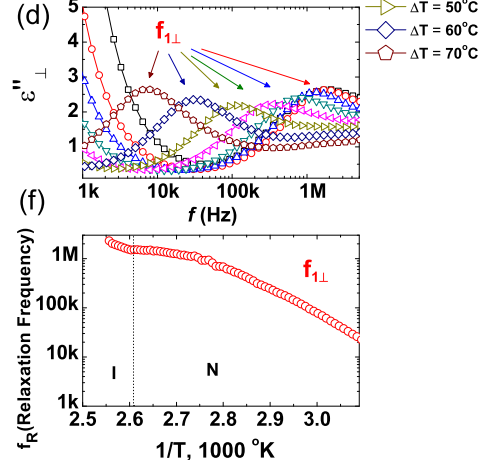

FIG. 2. (Color online) [(a) and (b)] Frequency dependence of the real parts of permittivity in the nematic phase of C7-BAN-CN with homeotropic and planar configuration for various reduced temperatures. (a) $\epsilon_{\|}^{\prime}$ and (b) $\epsilon_{\|}^{\prime}$ are the parallel and perpendicular components of the permittivity, respectively. (c) $\epsilon_{\|}^{\prime}, \epsilon_{\perp}^{\prime \prime}$ and (d) are the corresponding plots for dielectric loss. (e) $f_{1 \|}$ and (f) $f_{1 \perp}$ are the corresponding relaxation frequencies as functions of temperature; these are the frequencies for the maximum dielectric loss; the shape parameters of fitting are found as $\alpha, \beta>0.9$.

permittivity $\epsilon_{\|}^{\prime}$ and the loss $\epsilon_{\|}^{\prime \prime}$ are shown in Figs. 2(a) and 2(c), respectively, for various values of reduced temperature $\Delta \mathrm{T}=\mathrm{T}_{N I}-\mathrm{T}$. The perpendicular components of the permittivity $\epsilon_{\perp}^{\prime}$ and the loss $\epsilon_{\perp}^{\prime \prime}$ are given in Figs. 2(b) and 2(d). Here $\mathrm{T}_{N I}$ represents the isotropic to nematic transition temperature and $\mathrm{T}$ implies the temperature in degree celsius at which the measurement is carried out. In the low frequency range, $\boldsymbol{\epsilon}_{\perp}^{\prime}$ shows increase with a decrease in temperature. Meanwhile, $\epsilon_{\|}^{\prime}$ dramatically decreases with a reduction in temperature and ends up with falling below $\epsilon_{\perp}^{\prime}$. The temperature dependences of dielectric permittivities, $\boldsymbol{\epsilon}_{\perp}^{\prime}$ and $\boldsymbol{\epsilon}_{\|}^{\prime}$ are given in Fig. 3. Just below the transition temperature from the isotropic to nematic phase, sign reversal in $\Delta \epsilon^{\prime}$ is observed. However at relatively low temperatures, the material shows only negative $\Delta \epsilon^{\prime}$ regardless of the frequency of the applied field as shown in Figs. 3(a)-3(d) as $\boldsymbol{\epsilon}_{\|}^{\prime}$ drops below $\boldsymbol{\epsilon}_{\perp}^{\prime}$. Recently, Yoon $e t a l .^{3}$ have suggested that the two conformers of a bent-core LC may contribute to dual frequency $\left(f_{c}\right)$ response in different ranges of temperature.

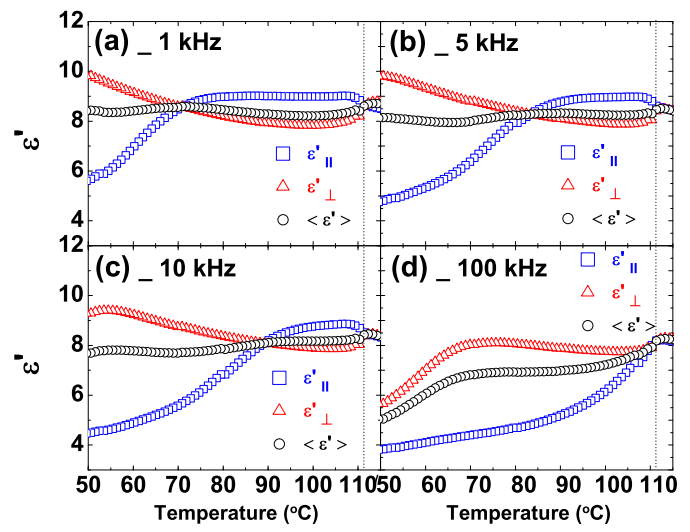

FIG. 3. (Color online) The dielectric permittivities at frequencies of 1, 5, 10, and $100 \mathrm{kHz}$ and $\left(\Delta \epsilon^{\prime}\right)=\left(\epsilon_{\|}^{\prime}-\epsilon_{\perp}^{\prime}\right)$ as functions of temperature. The average dielectric permittivity is calculated as $\left\langle\epsilon^{\prime}\right\rangle=\left(\epsilon_{\|}^{\prime}+2 \epsilon_{\perp}^{\prime}\right) / 3$.

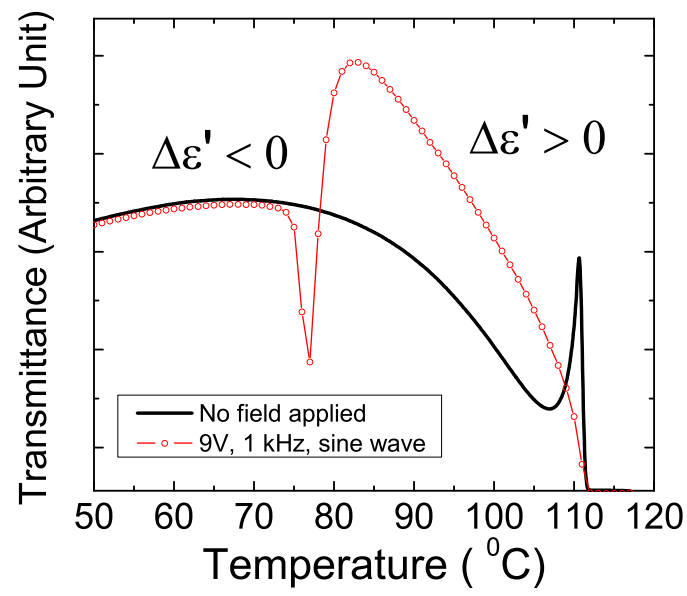

FIG. 4. (Color online) The transmittance curves for a planar cell with and without applied field, as indicated in the inset. Freederick's transition is observed at $72{ }^{\circ} \mathrm{C}$.

For a system where the number density of ions is very large, it is extremely difficult to carry out dielectric measurements. In such a case, an optical method is preferred. Furthermore in order to confirm the phenomenon of sign reversal in $\Delta \epsilon^{\prime}$ in the entire ranges of frequency and temperature, measurements using optical transmittance spectroscopy are carried out, where the in-phase and out of phase of the transmittance through the cell are measured. While the planar cell used in dielectric measurement is cooled from the isotropic to the nematic phase, the transmittance under crossed polarizers is recorded. In Fig. 4, two transmittance curves with and without electric field are plotted as a function of temperature. With an electric field at a frequency of $1 \mathrm{kHz}$ applied across the cell, the sample is heated from the nematic to the isotropic phase, the Freederick's transition is observed at a temperature of $72{ }^{\circ} \mathrm{C}$. Below this temperature, no Freederick's transition is observed for a field up to $2 \mathrm{~V} / \mu \mathrm{m}$ across the cell. Freederick's transition can only occur when the threshold field across the cell satisfies the equation $\mathrm{E}_{t h}$ $=(\pi / \mathrm{d}) \sqrt{\mathrm{K} / \epsilon_{o} \Delta \epsilon^{\prime}}$. Here $\mathrm{K}$ is the splay elastic constant and $\mathrm{d}$ is the cell gap. Normally it occurs above $\mathrm{T}_{c}$ where $\Delta \epsilon^{\prime}$ is positive and the field is slightly greater than the threshold field.

Measurements of the optical transmittance are carried out as a function of frequency for different temperatures. The sign reversal in $\Delta \epsilon^{\prime}$ is confirmed as a function of both temperature (crossover temperature) and the frequency. This is shown in Fig. 5. Interestingly $f_{c}$ versus temperature plot appears Arrhenius only over a very limited temperature range. This is feasible since $f_{c}$ does not involve a single activation process.

The static dielectric anisotropy, $\Delta \epsilon$, extrapolated to zero frequency in the nematic phase is given by the formula derived by Maier-Meier as follows: ${ }^{7}$

$$
\Delta \epsilon=\frac{\mathrm{N} h F}{\epsilon_{o}}\left[\Delta \alpha+\frac{F \mu^{2}\left(1-3 \cos ^{2} \beta\right)}{k \mathrm{~T}}\right] S
$$

Here $\mathrm{N}$ is the number density, $h$ and $F$ are the internal field factors, $\epsilon_{0}$ is the permittivity of vacuum, $\Delta \alpha$ is the anisotropy of the polarizability, $\mathrm{S}$ is the orientational order parameter, $k \mathrm{~T}$ is the thermal energy, and $\beta$ is the angle between the molecular net permanent dipole moment and the long molecular axis of the molecule. In this case the long axis is 


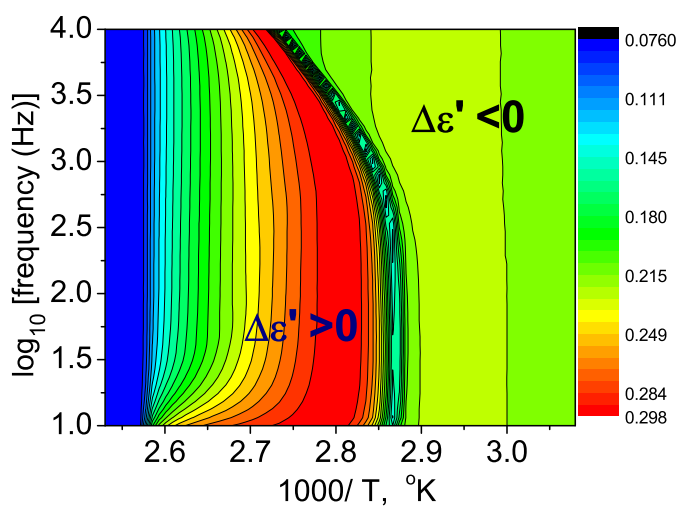

FIG. 5. (Color online) Frequency-temperature plot of the transmittance curves, the contour-line implies a constant value for the transmittance. Slope of the crowded curved lines $f_{c}$ as a function of $1 / \mathrm{T}$ appears Arrhenius only over a limited temperature range. Ordinate values on the right are colorcoded transmittances in arbitrary units.

defined as the axis joining the two end points of the banana molecule. In this expression $\beta$ governs the change in the sign of $\Delta \epsilon$. When $\beta$ is less than $54.7^{\circ}, \Delta \epsilon$ is negative. In general, $\beta$ is constant for a system in a nematic phase of calamitic molecules, and $\Delta \epsilon$ is governed by both $S$ and $T$. However, the LC dimers where the mesogens are linked by a flexible alkyl chain form a banana shaped molecule and these may show temperature dependence of $\beta$. Such a flexibility can give rise to both a change in $\beta$ and the magnitude of the molecular dipole moment as was found by Dunmur et al. ${ }^{8}$ The short range correlation between the molecular dipole moments can affect the static dielectric permittivity of nematic phase near transition to smectic phase. Following de Jeu et al., ${ }^{9}$ a change in the sign in the dielectric anisotropy on transition from nematic to smectic A arises from an enhanced dipole-dipole interactions prevalent in the molecules within layers.

The dipole-dipole interactions tend to decrease the dipole contributions to $\mu_{\|}$due to an increased dipole correlation in the smectic layers. As the distance between the molecules within a single smectic layer is much lower than the distance between the adjacent layers, an increased antiparallel correlation between the components of the dipole moments along the molecular long axis can be expected. de Jeu et al. through a detailed calculation have shown that the dipole moment $\mu_{\|}$is reduced, which in turn leads to a reduction in $\epsilon_{\|}$. At the same time, the dipole moment in the perpendicular direction $\mu_{\perp}$ is increased somewhat. In this context it means that the observation of the change in the sign of $\Delta \epsilon$ is a direct indication of the presence of cybotactic clusters with short range smectic order. The presence of these cybotactic groups as already stated is now supported by the x-ray diffraction studies through splitting of the x-ray pattern. ${ }^{6,10}$ Recently Vaupotič et al. ${ }^{11}$ showed that the splitting in the $\mathrm{x}$-ray pattern of the nematic phase is related to the SmC-like fluctuations when the lower-temperature phase is SmC. The splittings in the $\mathrm{x}$-ray pattern of C7-BAN-CN have similarly been interpreted in terms of SmC-like nanostructures. ${ }^{6}$

As shown in Figs. 2(c) and 2(d), two strong relaxations $\left(f_{\|}, f_{\perp}\right)$ are seen in the cells with homeotropic and planar configurations, respectively. It is interesting to note that the height of the dielectric loss peak in the planar configuration increases with a reduction in temperature whereas in the homeotropic configuration this stays almost constant. This can also be explained by the possibility of conformers where the bend angle decreases with an increase in temperature. The presence of such conformers is also supported by the NMR studies of Dong. ${ }^{5}$ The relaxation in the planar and homeotropic cells can be tentatively assigned to the rotation of the molecule around its long and short molecular axis, respectively. ${ }^{12}$ Recently Salamon et al. observed more relaxations at relatively lower frequencies in the dielectric spectra of a resorcinol derivative in its nematic phase than the conventional calamitic ones ${ }^{4}$ and some of these have been suggested to arise from a range of different conformers.

The sign reversal in the dielectric anisotropy is found to be partly due to a relatively large decrease in $\epsilon_{\|}^{\prime}$ and increase in $\epsilon_{\perp}^{\prime}$ with a reduction in temperature. A decrease in $\epsilon_{\|}^{\prime}$ and increase in $\epsilon_{\perp}^{\prime}$ with a reduction in temperature occurs due to cybotatic clusters. These may also occur partly from the conformers that become possible such that the net dipole moment parallel to long axis decreases and those normal to the long axis increases. For $f \geq f_{c}$, dielectric dispersion in $\epsilon_{\|}^{\prime}$ is almost negligible since $\epsilon_{\|}^{\prime}$ drops much below $\epsilon_{\perp}^{\prime}$. For this reason the dependence of the average permittivity at a frequency of $100 \mathrm{kHz}$ with temperature does not follow the normal trend.

In summary, we observe the sign reversal in $\Delta \epsilon^{\prime}$ in the nematic phase, for the first time such that at temperatures lower than $\mathrm{T}_{c}$, this anisotropy is independent of frequency. Moreover the cross-over frequency $f_{c}$ is highly nonArrhenious especially at lower temperatures. The observations are explained by the presence of nanocybotactic clusters as well by the presence of conformers that are allowed by flexible core of bent-core LC. These are supported by the existence of cybotactic clusters observed by $\mathrm{x}$-ray scattering as well as by the possibility of the conformers allowed by the rotation of the polar groups around bonds in the banana shaped molecule. This work needs to be extended further to find the relative contributions of each of the processes to the observations.

Grant Nos. EU-FP7-216025 BIND and SFI RFP 06/RFP/ ENE039 are acknowledged for funding.

${ }^{1}$ E. P. Raynes and I. A. Shanks, Electron. Lett. 10, 114 (1974).

${ }^{2}$ M. Schadt, Annu. Rev. Mater. Sci. 27, 305 (1997).

${ }^{3}$ H. G. Yoon, S.-W. Kang, R. Y. Dong, A. Marini, K. A. Suresh, M. Srinivasarao, and S. Kumar, Phys. Rev. E 81, 051706 (2010).

${ }^{4}$ P. Salamon, N. Eber, A. Buka, J. T. Gleeson, S. Sprunt, and A. Jakli, Phys. Rev. E 81, 031711 (2010).

${ }^{5}$ R. Y. Dong, J. Phys. Chem. B 113, 1933 (2009).

${ }^{6}$ C. Keith, A. Lehmann, U. Baumeister, M. Prehm, and C. Tschierske, Soft Matter 6, 1704 (2010).

${ }^{7}$ W. Maier and G. Meier, Z. Naturforsch. 16A, 262 (1961).

${ }^{8}$ D. A. Dunmur, G. R. Luckhurst, M. R. de la Fuente, S. Diez, and M. A. P. Jubindo, J. Chem. Phys. 115, 8681 (2001).

${ }^{9}$ W. H. de Jeu, W. J. A. Goossens, and P. Bordewijk, J. Chem. Phys. 61, 1985 (1974).

${ }^{10}$ A. G. Vanakaras and D. J. Photinos, J. Chem. Phys. 128, 154512 (2008)

${ }^{11}$ N. Vaupotič, J. Szydlowska, M. Salamonczyk, A. Kovarova, J. Svoboda, M. Osipov, D. Pociecha, and E. Gorecka, Phys. Rev. E 80, 030701(R) (2009).

${ }^{12}$ P. Tadapatri, U. S. Hiremath, C. V. Yelamaggad, and K. S. Krishmamurthy, J. Phys. Chem. B 114, 1745 (2010). 